\title{
Experimental observations of root growth in a controlled photoelastic granu- lar material
}

\author{
Jonathan Barés ${ }^{1, \star}$, Serge Mora ${ }^{1, \star \star}$, Jean-Yves Delenne ${ }^{2, \star \star \star}$, and Thierry Fourcaud ${ }^{3, \star \star \star \star}$ \\ ${ }^{1}$ Laboratoire de Mécanique et Génie Civil, Université de Montpellier, CNRS, Montpellier, France \\ ${ }^{2}$ UMR IATE, CIRAD, INRA, Montpellier SupAgro, Université de Montpellier, Montpellier, France \\ ${ }^{3}$ CIRAD, UMR AMAP, Montpellier, France
}

\begin{abstract}
.
We present a novel root observation apparatus capable of measuring the mechanical evolution of both the root network and the surrounding granular medium. The apparatus consists of 11 parallel growth frames, two of them being shearable, where the roots grow inside a photo-elastic or glass granular medium sandwiched between two pieces of glass. An automated system waters the plant and image each frame periodically in white light and between crossed polarisers. This makes it possible to follow (i) the root tips and (ii) the grain displacements as well as (iii) their inner pressure. We show how a root networks evolve in a granular medium and how it can mechanically stabilize it. This constitutes a model experiment to move forward in the understanding of the complex interaction between root growth and surrounding soil mechanical evolution.
\end{abstract}

\section{Introduction}

When growing, plants and trees adapt their morphology according to the mechanical and topological properties of the surrounding soil. Conversely, they change the mechanical and chemical properties of this environment [1]. Depending on the soil density or confining pressure, on the size and architecture of the voids between solid constituents of the soil, the roots will be blocked or grow farther [2-4], either by curving, changing their direction or by stressing the soil material $[5,6]$. Therefore, the competing effects of soil mechanical impedance (mechanical property) [7] and penetrating capacities of the plant (biological property) lead to different strategies for the root development which can be optimized, and which in turn modifies differently the soil physical properties. Indeed, the root system architecture (RSA) impacts plant fitness and crop yield by facilitating efficient nutrient and water uptake from the soil. A better understanding of the effects of soil on RSA could improve crop productivity by matching roots to their soil environment [8]. Conversely, roots can change soil aeration, local packing fraction and constituent organization $[9,10]$ but also its repartition of water and cohesion $[11,12]$. Hence, the mechanism of root growth in soil is a key issue to understand both how to improve plant development and how to improve grounds properties like stability for example. When a plant grows its roots have an effect on the stress development in the surrounding soil

\footnotetext{
^e-mail: jb@jonathan-bares.eu

$\star \star$ e-mail: serge.mora@umontpellier.fr

$\star \star \star$ e-mail: jean-yves.delenne@supagro.inra.fr

$\star \star \star \star$ e-mail: thierry.fourcaud@cirad.fr
}

considered as a granular material. Conversely the induced pressure of the granular medium on the roots can affect the root network growth. We want to understand this complex root-grains, biological and mechanical, feedback loop between plant roots and soil structure and composition [13].

At the local scale, [5, 6] measured the force applied by a growing root tip on an obstacle. At the same scale, [1] have also studied the stress induced by a root when it goes through the gap between 2 bidimensional photoelastic grains. At the global scale [14] attempted to observe the root network in a packing of such particles. But root tip cannot initiate a path if there is no gap between grains, which is the case for a 2D dense packing of cylinders. From a numerical perspective, [11, 15] have modelled root network architectures in different soils while [16-18] have studied the tree anchorage and soil stabilization phenomenons.

Still, no experimental studies have been carried out to directly observe root network dynamics and surrounding stress modification while imposing specific grain configurations and density or specific mechanical loadings. It is therefore of primary importance to design a model experiment to understand progressively the intricate soil-root system from a mechanical point of view [1]. In this paper, we present a novel set-up which permits to observe the development of chickpea (cicer arietinum L.) root networks in a 2D granular material constituted of $(i)$ home made bidisperse photoelastic discs or of (ii) glass beads. The photo-elastic particles $(i)$ are molded with a groove on their edges so that the root tips are able to initiate a path between grains. Nevertheless the groove is small enough to observe a photoelastic signal when the root get thicker. 
The position of the grains, the inter-granular spacing as well as the nature of the system confinement can be imposed in this set-up. The container of the granular material can be (i) an open cell, (ii) a confined cell and even a (iii) shearable cell. In this last case a slow shear loading can be imposed during the root growth or at the end of the development to test the stabilization effect on the granular medium. In the automated experimental apparatus several root development cells are treated in parallel to increase the statistical meaning of the observations. For each cell, each hour a picture is taken with and without polarized light. Then the evolution of the root network is followed as well as the position and pressure inside each particle by mean of classical image processing [19] and photo-elastic techniques [20, 21].

\section{Experimental set-up}

\subsection{Growth frames}

In the experimental set-up we observe the growth of chickpea (cicer arietinum L.) root networks in quasi-2D granular materials. Chickpea has been chosen because it is robust, the thickness of the roots $(\sim 1 \mathrm{~mm})$ is about the depth of the $2 \mathrm{D}$ medium $(4 \mathrm{~mm})$, the root network is not too dense, it grows relatively rapidly (few weeks) and the seed is large enough to feed the plant during the first cycle of the growth. In a first configuration, these chickpeas are grown in simple $15 \mathrm{~cm}^{2}$ cells between 2 pieces of glass separated by $4 \mathrm{~mm}$, as presented in fig. 1-A. In a second configuration, as presented in fig.1-B the left and write edges of the cell are movable so that the whole system (grains and roots) can be sheared by mean of a stepper motor once the root network is fully developed. In both case the plants grow in deionized water.

As shown in fig.1-A, to observe the growth, periodically (each hour) the cells are lit from the back with a flat circularly polarized light and imaged from the front with a 20Mp CCD camera ${ }^{1}$ with and without a crossed polariser in front of the camera length. To observe the growth of several plants at a time, in several configurations, and to increase the statistical representativity of our results, 11 cells are observed at the same time ( 2 of them being shearable). As presented in fig.1-C the growth frames lay on a bar in parallel and the imaging part of the device, moved by a stepper motor, slides in front of each cell to take pictures. The whole system, including the light control is automated as well as a watering system which maintain the deionized water level constant in each cell (not shown here).

\subsection{Granular material}

These chickpeas are grown either in bidisperse photoelastic discs ( 7 and $9 \mathrm{~mm}$ in diameter) or in bidisperse glass beads ( 2 and $4 \mathrm{~mm}$ in diameter). In the first case to avoid the problem with cylindrical particles pointed out by [14], as presented in fig.2-C, we used particles with a $0.5 \mathrm{~mm}^{2}$
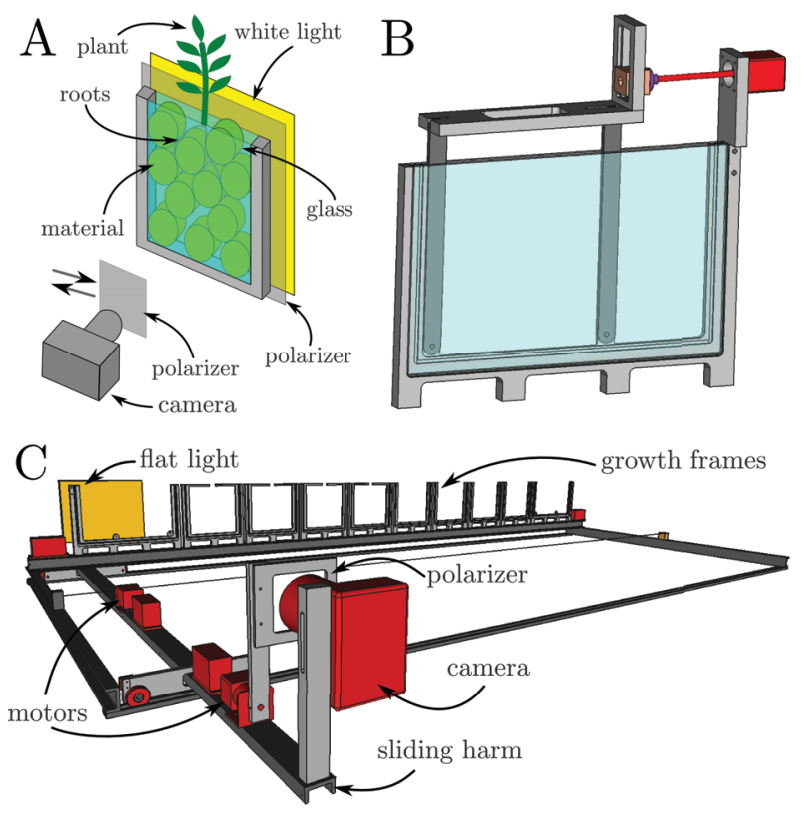

Figure 1. Experimental set-up. A: Imaging principle. The plant growths sandwiched between two pieces of glass in a $4 \mathrm{~mm}$ thick flat frame with $15 \mathrm{~cm}$ width and $15 \mathrm{~cm}$ height. The roots growth in a $2 D$ granular material constituted of bidisperse glass beads or photo-elastic cylinders covered with deionized water. Periodically, the system is imaged by a front camera with a back-light with and without cross polarisers. B: Shearable growth frame. Some plants have been grown in frames equipped with a parallelogram mechanism to shear the granular medium when the growth is ended. The mechanism is actuated by a stepper motor. C: Complete set-up. The whole set-up is constituted of 11 growth frame (2 shearable, 9 simple) laying in parallel. Periodically a robotic harm slides in front of each frame to image it with and without polariser. Lighting is also controlled for pictures.

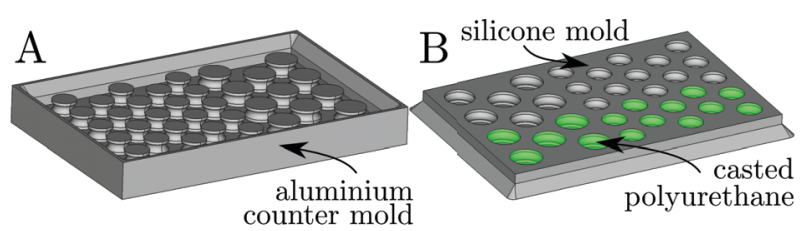

C

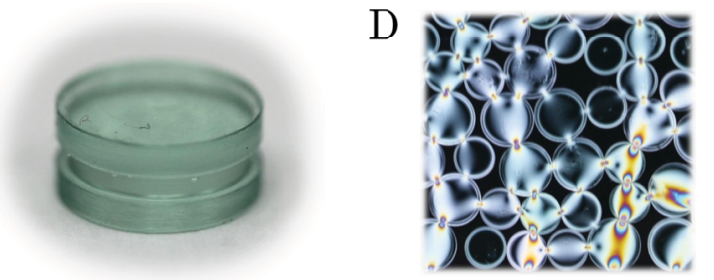

Figure 2. Photo-elastic grain molding. A: Aluminium counter mold of a set of 34 bidisperse particles. Particles are $4 \mathrm{~mm}$ thick cylinders of diameter $7 \mathrm{~mm}$ and $9 \mathrm{~mm}$ with a $0.5 \mathrm{~mm}$ deep and $1 \mathrm{~mm}$ wide groove on the edge (see C). B: Silicone mold made from the aluminium counter mold. Colored polyurethane is molded in each alveolus. C: Photo-elastic polyurethane particle. D: Collection of compressed particles imaged between circular cross polarisers. Colored regions of the particles shows highly stressed areas. 

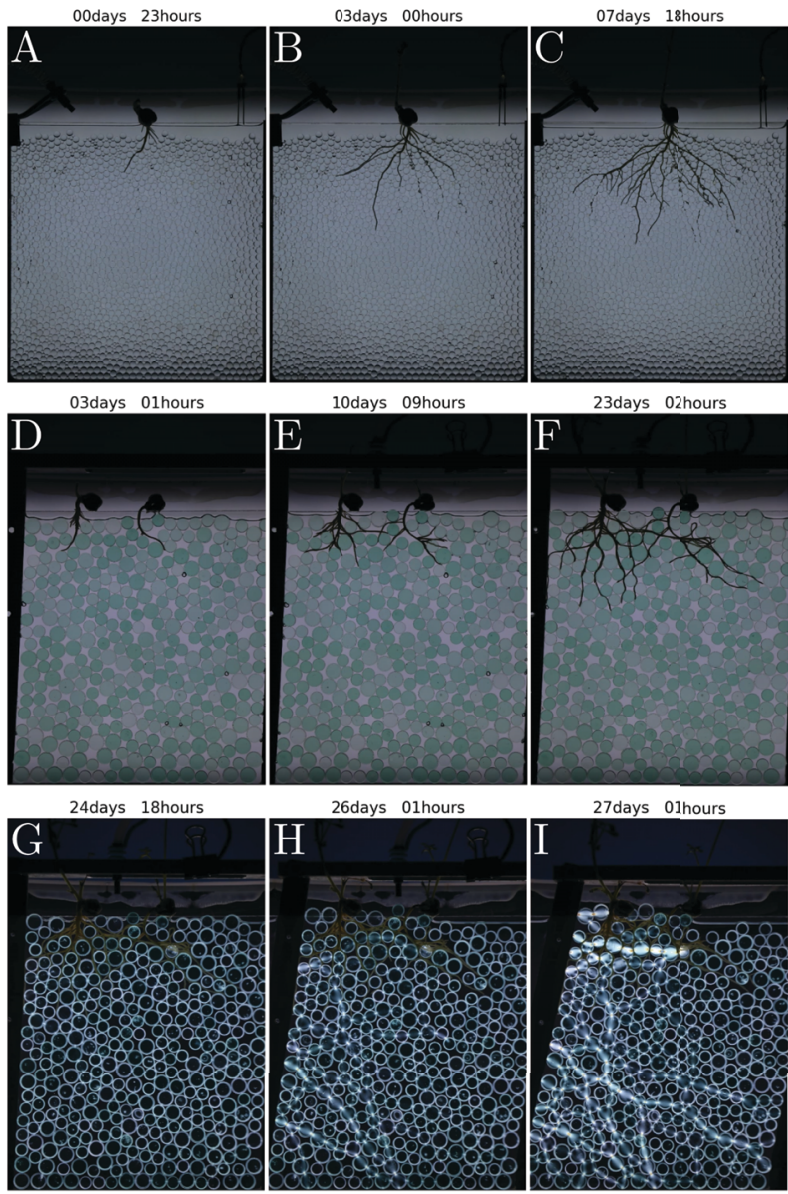

Figure 3. A, B, C: Development of a chickpea root network $c i$ cer arietinum $L$. in a bi-disperse quasi-monolayer of glass beads ( 2 and $4 \mathrm{~mm}$ diameters). After a the main root network growth (A and B), a secondary one develops (C). Root oscillates betweens the beads. C, D, and E: Development of two chickpea root networks in a bi-disperse layer of photo-elastic particles. G, H and I: The system is then sheared quasistatically. The root networks avoid the top grains to rearrange and make the granular medium jammed. For A, B, C, D, E and F pictures are taken only with back-lighting while for pictures $\mathrm{G}, \mathrm{H}$ and I the system is between crossed polarisers.

groove on the edge. Hence root tip can initiate a way between two adjacent grains and propagate deep inside the granular medium. These grooved photo-elastic particles are molded from polyurethane using the technique first introduced by [19]. As shown in fig.2-A a counter mold is machined out of aluminium. Then, a mold is made from silicon rubber ${ }^{2}$ (see fig.2-B) and colored polyurethane ${ }^{3}$ is molded in each hole. As shown in fig.2-D the photoelasticity of these particles is very good and classical techniques for photo-elastic granular materials pioneered by $[20,21]$ can by used.

\section{Results and discussions}

In fig.3-A, B and $\mathrm{C}$ we show the development of a chickpea root network in a bi-disperse quasi-monolayer of glass beads. After one day, the main root is already embedded in the granular material and secondary roots appear at the basis of the seed. Two days later, the full main root network is set and has moved the grains around the seed. After one week, the RSA is at its maximum of expansion without more nutriment. Secondary roots have grown from the main network and we clearly observe the root tortuosity imposed by the granular arrangement. Experiment is stopped and root dynamics can be studied.

In fig.3-D, E and F we show the development of two chickpea root networks in a bi-disperse layer of photoelastic particles. Compared to the glass bead case the growth is much slower and plants need to be fed: deionized water is oxygenated and enriched. In this case it takes more then 3 weeks for the RSA to be fully developed. Tortuosity is also qualitatively lower since grains are larger than glass beads. After 23 days, the system is slowly and continuously sheared of $21 \%$ during 4 days. During this process even if the granular system is opened on its top and free to rearrange, a strong force network builds up. We see in fig. 3-G, H and I that the root networks prevent the grains from rearranging and releasing stress. This create force chains forming from top left to the bottom right of the cell and from the left to the roots. This shows how the chickpea RSA can stabilize a granular system.

As presented in fig.3-A to $\mathrm{F}$ the back light pictures permit to observe the root network with a high contrast. This makes the detection of the RSA easy by thresholding the pictures. Hence, fig.4-A shows the position of the root network between photo-elastic grains once the RSA is fully developed. Just like already done in gels by [22], a homemade code as been written to detect and track the root tips during the development of the RSA. Blue and red lines in the figure are two examples of the path followed by two root tips. In both cases, we observe that the path does not follow the final root architecture. This means the root widely rearranges during its growing process either due to granular displacement or to the growing of secondary roots. Root-grain interaction is thus a dynamical process which needs to be followed on the long term. From the evolution of the root tip position, in fig.4-C, we plot the root speed for the two selected roots of the two plants. We see that the growth speed is not constant and not equivalent for both plants. However, it is difficult to analyse if this speed variation is due to biological or mechanical parameters. We also observed root tip circumnutation [23] in 2D (not shown here), how it is affected by the surrounding granular medium, how it changes the granular local organization and how it creates the root tortuosity.

Finally, from the back light pictures (see fig.3-F for instance), using image processing tools designed by [19], we detect the grain positions and sizes, and from the polarized pictures (see fig.3-I for instance) we compute the

\footnotetext{
${ }^{2}$ Smooth-On, MoldStar ${ }^{\circledR} 15$ (slow).

${ }^{3}$ Smooth-On, ClearFlex ${ }^{\circledR} 50$ with green SoStrong ${ }^{\circledR}$ ink.
} 

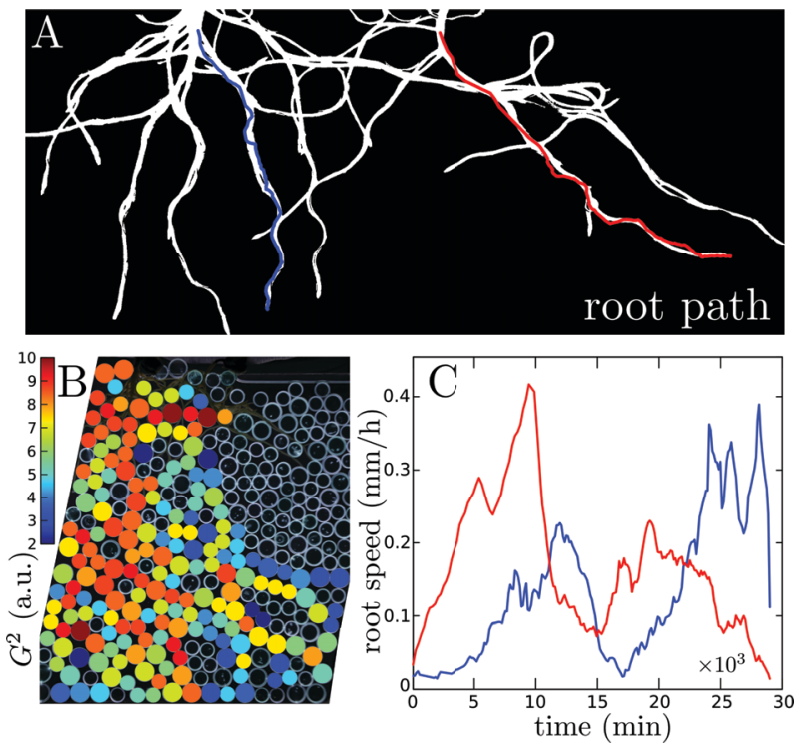

Figure 4. A: Root tip path. The black and white back ground shows the root networks of two chickpeas grown in photo-elastic discs just before the system is sheared. Blue and red line shows the path followed by two root tips (arbitrarily chosen). We see that due to the rearrangement of the root and grains the final root does not follow exactly the root tip path. B: Pressure measurement. For the maximum strain, when the root networks are fully developed, grain position has been tracked and pressure measured in each of them as $G^{2}$ [20]. By sake of clarity, grains with pressure lower than a certain value (2) are not presented. The force chains show the stabilization effect of the root networks $C$ : Root tip speed. For root tips presented in blue en red in A, the evolution of their speed is shown during the plant growth before shearing the system. We see that the growth speed is not constant and not equivalent for both plants.

grain pressures in terms of $G^{2}$ as explained in [19-21]. For a system with a RSA fully developed and $21 \%$ sheared, fig.4-B shows the pressure in each grains. We observe force chains and measure the highest pressure near the root network. This is another observation of the fact that the RSA stabilizes the granular system.

\section{Conclusion}

We have described a novel experimental system that can observe the growth of chickpea root networks in different granular materials (namely grooved photo-elastic discs and glass beads) for different system confinements (namely open frames and sheared frames). The growth is observed both in white light and between crossed polarisers. This permits to measure the evolution of the RSA and more specifically to follow the root tips. The grain rearrangements and the evolution of their inner pressure is also recorded. This constitutes a model experiment to move forward in the understanding of the complex interaction between root growth and surrounding soil mechanical evolution.

\section{References}

[1] E. Kolb, C. Hartmann, P. Genet, Plant and Soil 360, 19 (2012)

[2] A.M. Abdalla, D.R.P. Hettiaratchi, A.R. Reece, Journal of Agricultural Engineering Research 14, 236 (1969)

[3] L.J. Clark, W.R. Whalley, P.B. Barraclough, in Roots: The Dynamic Interface Between Plants and the Earth (Springer, 2003), pp. 93-104

[4] S.R. Tracy, C.R. Black, J.A. Roberts, S.J. Mooney, Journal of the Science of Food and Agriculture 91, 1528 (2011)

[5] R.K. Misra, A.R. Dexter, A.M. Alston, Plant and Soil 95, 315 (1986)

[6] A.G. Bengough, C.J. Mackenzie, H.E. Elangwe, Plant and Soil 167, 135 (1994)

[7] B.J. Atwell, Environmental and Experimental Botany 33, 27 (1993)

[8] E.D. Rogers, D. Monaenkova, M. Mijar, A. Nori, D.I. Goldman, P.N. Benfey, Plant physiology pp. pp00397 (2016)

[9] H.P. Cresswell, J.A. Kirkegaard, Soil Research 33, 221 (1995)

[10] S.A. Materechera, A.R. Dexter, A. A. M. Alston, Plant and Soil 142, 69 (1992)

[11] S. Baets, D. Torri, J. Poesen, M.P. Salvador, J. Meersmans, Earth Surface Processes and Landforms 33, 1948 (2008)

[12] F. Danjon, D.H. Barker, M. Drexhage, A. Stokes, Annals of Botany 101, 1281 (2008)

[13] A. Pierret, C. Doussan, Y. Capowiez, F. Bastardie, Vadose Zone Journal 6, 269 (2007)

[14] D.M. Wendell, K. Luginbuhl, J. Guerrero, A.E. Hosoi, Experimental Mechanics 52, 945 (2012)

[15] M. Heinen, A. Mollier, P.D. Willigen, Plant and Soil 252, 251 (2003)

[16] L.X. Dupuy, T. Fourcaud, P. Lac, A. Stokes, American Journal of Botany 94, 1506 (2007)

[17] A. Stokes, C. Atger, A.G. Bengough, T. Fourcaud, R.C. Sidle, Plant and Soil 324, 1 (2009)

[18] S.B. Mickovski, A. Stokes, R. van Beek, M. Ghestem, T. Fourcaud, Ecological Engineering 37, 1523 (2011)

[19] M. Cox, D. Wang, J. Barés, R.P. Behringer, Europhysics Letters 115, 64003 (2016)

[20] D. Howell, R.P. Behringer, C. Veje, Physical Review Letters 82, 5241 (1999)

[21] T.S. Majmudar, R.P. Behringer, Nature 435, 1079 (2005)

[22] M.V. Thompson, N.M. Holbrook, Plant Physiology 135, 1822 (2004)

[23] M. Stolarz, Plant Signaling \& Behavior 4, 380 (2009), pMID: 19816110, http://dx.doi.org/10.4161/psb.4.5.8293 